\title{
Correction to: Influence of Body Odors and Gender on Perceived Genital Arousal
}

\author{
Patrícia Alves-Oliveira $^{1,9}$ (1) $\cdot$ Joana Carvalho ${ }^{2,3} \cdot$ Jacqueline Ferreira $^{4,5,6}$. \\ Laura Alho $^{2,3}$ - Pedro Nobre ${ }^{7} \cdot$ Mats J. Olsson $^{8}$ - Sandra C. Soares ${ }^{4,5,8,10}$
}

Published online: 12 February 2018

(C) Springer Science+Business Media, LLC, part of Springer Nature 2018

\section{Correction to: Arch Sex Behav https://doi.org/10.1007/s10508-017- 1091-5}

There were 4 cells in the original Table 2 that reported SEM values instead of SDs. A corrected version of Table 2 is provided below. In the Results section, the corresponding corrections are as follows:
1. For Odor Effects by Gender (for Women): In the second paragraph, 5th line: The correct $\mathrm{SD}=2.17$. On the 6th line, the correct $\mathrm{SD}=1.88$.

2. For Odor Effects by Gender (for Men): In the first paragraph, 5th line: The correct $\mathrm{SD}=2.78$. On the 6th line, the correct $\mathrm{SD}=2.30$.

The seven authors of the paper apologize for these errors.

The original article can be found online at https://doi.org/10.1007/ s10508-017-1091-5.

Joana Carvalho

joana.pereira.carvalho@ulusofona.pt

1 Instituto Universitário de Lisboa (ISCTE-IUL), CIS-IUL, 1600-077 Lisbon, Portugal

2 Escola de Psicologia e Ciências da Vida, Universidade Lusófona de Humanidades e Tecnologias, Lisbon, Portugal

3 Centro de Psicologia da Universidade do Porto, Porto, Portugal

4 Department of Education and Psychology, University of Aveiro, Aveiro, Portugal

5 Center for Health Technology and Services Research, Faculty of Medicine, University of Porto, Porto, Portugal

6 Institute for Biomedical Imaging and Life Sciences, Faculty of Medicine, University of Coimbra, Coimbra, Portugal

7 Faculdade de Psicologia e Ciências da Educação, Universidade do Porto, Porto, Portugal

8 Division of Psychology, Department of Clinical Neuroscience, Karolinska Institute, Solna, Sweden

9 Instituto de Engenharia de Sistemas e Computadores Investigação e Desenvolvimento, Porto Salvo, Portugal

10 William James Center for Research, ISPA University Institute, Lisbon, Portugal 
Table 2 Descriptive statistics of subjective odor ratings (intensity, pleasantness, familiarity) and perceived genital arousal for women and men, including one-way ANOVA results and significance level

\begin{tabular}{|c|c|c|c|c|c|c|}
\hline & & & $\begin{array}{l}\text { Body odor condition } \\
M \text { (SD) }\end{array}$ & $\begin{array}{l}\text { No odor condition } \\
M \text { (SD) }\end{array}$ & One-way ANOVA & $p$ \\
\hline \multirow[t]{4}{*}{ Women } & \multirow[t]{3}{*}{ Subjective odor ratings } & Intensity & $5.33 \pm 2.35$ & $3.11 \pm 1.82$ & $F(1,38)=11.04$ & .002 \\
\hline & & Pleasantness & $3.62 \pm 1.66$ & $5.26 \pm 1.52$ & $F(1,38)=10.60$ & .002 \\
\hline & & Familiarity & $4.67 \pm 2.46$ & $3.11 \pm 1.85$ & $F(1,38)=5.06$ & .030 \\
\hline & Perceived genital arousal & & $1.86 \pm 1.88$ & $4.53 \pm 2.17$ & $F(1,38)=17.39$ & $<.001$ \\
\hline \multirow[t]{4}{*}{ Men } & \multirow[t]{3}{*}{ Subjective odor ratings } & Intensity & $5.26 \pm 1.82$ & $5.05 \pm 2.13$ & $F(1,38)=0.12$ & .734 \\
\hline & & Pleasantness & $5.84 \pm 1.74$ & $5.71 \pm 1.45$ & $F(1,38)=0.06$ & .802 \\
\hline & & Familiarity & $5.00 \pm 1.67$ & $4.05 \pm 2.20$ & $F(1,38)=2.34$ & .134 \\
\hline & Perceived genital arousal & & $3.58 \pm 2.30$ & $3.62 \pm 2.78$ & $F(1,38)=0.00$ & .961 \\
\hline
\end{tabular}

